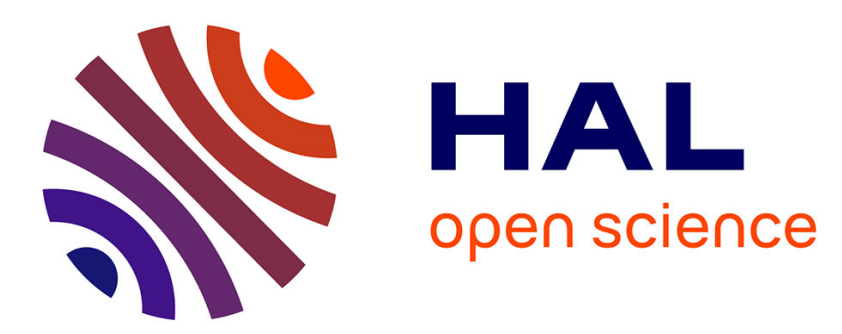

\title{
Jour et nuit: une géographie inversée de la pratique des quais à Bordeaux
}

Cécilia Comelli

\section{To cite this version:}

Cécilia Comelli. Jour et nuit: une géographie inversée de la pratique des quais à Bordeaux. Culture and Local Governance / Culture et gouvernance locale, 2010, 2 (2), https://uottawa.scholarsportal.info/ojs/index.php/clg-cgl/article/view/142. 10.18192/clgcgl.v2i2.142 . hal-00991095

\section{HAL Id: hal-00991095 \\ https://hal.science/hal-00991095}

Submitted on 2 Jul 2014

HAL is a multi-disciplinary open access archive for the deposit and dissemination of scientific research documents, whether they are published or not. The documents may come from teaching and research institutions in France or abroad, or from public or private research centers.
L'archive ouverte pluridisciplinaire HAL, est destinée au dépôt et à la diffusion de documents scientifiques de niveau recherche, publiés ou non, émanant des établissements d'enseignement et de recherche français ou étrangers, des laboratoires publics ou privés. 


\title{
CULTURE AND LOCAL GOVERNANCE CULTURE ET GOUVERNANCE LOCALE
}

\section{Jour et nuit : une géographie inversée de la pratique des quais à Bordeaux}

\author{
Comelli Cécilia ${ }^{1}$ \\ Université Bordeaux 3
}

\begin{abstract}
Résumé
La ville de Bordeaux est en pleine mutation depuis une dizaine d'année sous l'impulsion de son maire, Alain Juppé, élu en 1995. De nombreux chantiers ont vu le jour, les plus importants étant ceux de la mise en place du tramway et de la rénovation des quais. Ces derniers sont bordés par plusieurs kilomètres de façades XVIIlème, faisant la fierté de la ville et ayant participé à l'inscription de Bordeaux au patrimoine mondial de l'UNESCO. Cette mise en valeur du patrimoine, idéologie contemporaine des sociétés occidentales, appliquée aux quais de Bordeaux, a fait de cette façade une vitrine de la ville. Parmi les conséquences urbaines de ces rénovations on peut constater que la requalification de ces lieux, par l'attribution de nouvelles fonctions, a favorisé l'émergence de secteurs d'excellence allant de pair avec une certaine relégation tant sociale que spatiale. Les quais se révèlent alors fonctionner tel un système socio-spatial dont l'attractivité et la polarisation s'inversent entre le jour et la nuit.
\end{abstract}

\footnotetext{
${ }^{1}$ Doctorante en géographie, UMR 5185, ADES-CNRS, Université de Bordeaux 3.
} 


\section{Jour et nuit : une géographie inversée de la pratique des quais à Bordeaux}

\section{Introduction}

Lorsqu'il s'agit d'étudier une ville, la dimension temporelle est très rarement prise en compte. Peu de recherches traitent de cet aspect, à croire que la ville n'a qu'une facette, celle du jour. Le géographe Luc Gwiazdzinski regrette que la plupart des maires et des urbanistes pensent la ville comme si elle s'arrêtait à 19 heures. Qu'en est-il de Bordeaux qui est actuellement l'objet d'un grand projet de renouvellement urbain ? Pour répondre à cette question nous nous intéresserons aux quais de la ville dont les travaux sont en passe d'être terminés. Nous avons choisi le secteur des quais car cette partie de la ville a été l'objet d'une attention particulière de la part des aménageurs et qu'elle est caractéristique d'une rénovation plus globale de la ville de Bordeaux. Nous les étudierons dans une temporalité complète, en tenant compte du jour et de la nuit, et en traitant des conséquences urbaines de la mondialisation à travers la politique de la ville. Nous allons donc nous interroger sur l'incidence de la mondialisation dans l'espace public, de manière générale puis plus spécifique à Bordeaux, en nous demandant notamment ce qu'il en est des conséquences bien connues mais aussi de celles moins avouées.

Nous verrons tout d'abord que ces rénovations ne sont pas uniques à Bordeaux mais correspondent aux grandes idéologies contemporaines de notre aire culturelle occidentale en matière de patrimonialisation, c'est en cela que la mondialisation joue un rôle de vecteur en véhiculant cette idéologie. Nous nous attacherons à voir plus spécifiquement pour la ville de Bordeaux quelles sont ces rénovations, comment elles s'appliquent et quelles en sont les conséquences sur les fonctions des lieux et les pratiques des usagers.

Les quais étant dans un état d'abandon relatif avant les travaux, il est possible de s'interroger sur les effets d'une hausse des prix du foncier, nous verrons donc ensuite les conséquences de l'émergence de secteurs d'excellence, allant de pair avec une certaine relégation. Constate-t-on un accès discriminant aux nouvelles pratiques de ce lieu et notamment aux pratiques nocturnes ?

Enfin, Un autre aspect des quais sera abordé, celui d'un axe construit comme un système spatial où il est possible d'appliquer le modèle centre-périphérie. Nous verrons alors qu'une des particularités des quais a lieu la nuit et se traduit par l'inversion de ce modèle.

\section{1- L'HOMOGENEISATION DE L'ESPACE PUBLIC}

\subsection{La patrimonialisation : une idéologie contemporaine}

La rue est l'un des éléments de construction de l'identité d'une ville la rendant plus ou moins compétitive avec d'autres villes ayant développées des politiques proches. La "monumentalisation et la patrimonialisation par des restaurations 
immobilières ou des réalisations architecturales contemporaines jouent aujourd'hui un rôle décisif dans le processus d'internationalisation des villes "(Grafmeyer, 1994). La sauvegarde du patrimoine, par sa réhabilitation et sa mise en valeur, fait partie des grandes idéologies contemporaines en matière d'urbanisme et de politique de la ville. En effet, différentes lois soutiennent la mise en valeur du patrimoine, en particulier la loi Malraux de 1962 qui permet de protéger des secteurs urbains caractéristiques et l'instauration des ZPPAUP ${ }^{2}$ en 1983 afin de sauvegarder le patrimoine architectural urbain. A l'échelle internationale, la liste du patrimoine mondial établie par l'UNESCO date de 1972 et un Réseau européen du patrimoine existe depuis 1996. Mais c'est surtout depuis une dizaine d'années que l'on assiste, en Europe, à un " retour en ville », des populations et des politiques publiques (Bidou-Zachariasen, 2003).

La rénovation des quais a été menée dans cet état d'esprit de la patrimonialisation, qui n'est en rien spécifique à Bordeaux. La mondialisation a pour conséquence une standardisation, une homogénéisation croissante des paysages et des pratiques urbaines. La plupart des grandes villes européennes en rénovation le sont d'une manière similaire (Bernié-Boissard, 2008). Cette mise en conformité sous tendue par un imaginaire éco-sanitaire a pour effet « d'aseptiser » les villes afin de les rendre plus attrayantes. L'image d'une ville étant, entre autres, véhiculée par ses rues, ce sont ces dernières qui doivent renvoyer un sentiment de propreté, de sûreté. Cette " aseptisation " passe donc par un nettoyage des rues, au sens propre comme au figuré. Au sens propre, un nettoyage matériel par le ravalement des façades, la rénovation du bâti et l'aménagement général de la voirie, avec une tendance à la privatisation des espaces publics et à la conception de la ville comme un espace de consommation (Gasnier, 2006). Au sens figuré, un nettoyage " humain » plus ou moins direct est mis en œuvre dans de nombreuses villes. Il s'agit dans ce cas de repousser la précarité et l'insécurité hors du visible (notamment les SDF et les prostituées). C'est pour cela que « les espaces publics font l'objet de restrictions et de contrôles de plus en plus poussés » (Gasnier, 2006). Les villes ont différents moyens à leur disposition comme l'installation de caméras de surveillance, la régulation policière ou l'installation d'un mobilier urbain "inhumain » et inhospitalier (par exemple des bancs inconfortables) ${ }^{3}$. Ces pratiques de nettoyage matériel et humain contribuent à la mise en valeur et à l'attractivité des quais et donc à la hausse des prix du foncier.

Ces mesures concourent à une standardisation des espaces publics mais également des activités et des usagers de ces lieux. Les individus font ce qu'il leur est proposé : du vélo sur les pistes cyclables, des achats dans les hangars réhabilités en commerces... l'improvisation et l'imprévu est mal toléré (Sennet, 1990). La nuit est elle aussi marquée par cette standardisation. On assiste, d'après J.M. Deleuil (1994), à une homogénéisation de la nuit, des types d'établissement et de la population, cette normalisation des comportements rejaillit sur l'identité des quartiers. Ces derniers sont mis en valeur à travers les rues qui les composent, et bien qu'elles aient tendance à se ressembler, l'esthétique des rues (Roulleau-Berger, 2004) est devenue un enjeu politique. Enjeu au niveau local afin

\footnotetext{
${ }^{2}$ Zones de protection du patrimoine architectural, urbain et paysager.

${ }^{3}$ Voir à ce propos les travaux de Noël Jouenne.
} 
de rendre un quartier plus attractif pour des couches sociales susceptibles de dynamiser une économie urbaine par leur pouvoir d'achat élevé, mais c'est aussi un enjeu au niveau mondial, ainsi, certaines rues sont particulièrement mises en valeur pour devenir des symboles et servir de vitrine au rayonnement international de la ville.

Suivant cette idéologie et des directives semblables, les villes occidentales n'ont d'autres choix que de se ressembler mais doivent, paradoxalement, répondre à ces critères communs de valeurs et d'esthétique pour se différencier. Ce phénomène est apparu bien plus tôt aux Etats-Unis car dès les années 1960 Jane Jacobs a participé à diffuser une image positive des quartiers populaires anciens. Ce qui est à l'origine du processus que Ruth Glass qualifiera de gentrification en 1963.

\subsection{L’application de cette idéologie aux quais de Bordeaux}

Depuis une dizaine d'années sous l'impulsion d'Alain Juppé, élu maire de Bordeaux en 1995, la ville est en pleine mutation. Les quartiers anciens du centre sont rénovés et une attention particulière a été portée à la rénovation des quais. Ces derniers, très vivants durant des siècles grâce à l'activité portuaire, ont subi la désaffectation des équipements du port autonome de Bordeaux depuis les années 1980 : les activités ont été progressivement déplacées en aval pour quitter définitivement le centre ville en 1987. Le déplacement de ces activités a laissé les quais en friche, les hangars à l'abandon et les bords de l'eau inaccessibles derrière des kilomètres de grille. Durant cette période, les quais ont connu une désertification sur les deux rives, ainsi qu'une paupérisation et une hausse de l'insécurité, ces différents éléments concourant à véhiculer une image négative des quartiers adjacents (Programme opérationnel Unicités-urban2, 2001). Depuis 1997, différents programmes de réhabilitation se sont succédés ${ }^{4}$.

Toute personne ayant connu Bordeaux, et en particulier les quais, dans les années 1990 ne peut que constater les impressionnants changements qui ont eu lieu. Les quais ne sont plus désertés, ils ne véhiculent plus une image négative. Le pari est réussi pour la mairie de Bordeaux qui avait anticipé la transformation en attribuant de nouvelles fonctions à ces espaces. Ce lieu est devenu accueillant, les familles viennent s'y promener durant la journée et jeunes et moins jeunes s'y retrouver le soir quand les beaux jours arrivent. Le secteur a par ailleurs été classé au patrimoine mondial de l'humanité par l'UNESCO en 2007. Les quais nous paraissent être caractéristiques de la "mise au propre " de la ville à Bordeaux. En effet, en quelques années le paysage et la morphologie des bords de Garonne ont considérablement changés et cet axe, longtemps délaissé, est devenu un secteur d'excellence au sein de la ville. Ces changements réalisés suivant les plans du paysagiste-urbaniste Michel Courajoud ont surtout eu lieu

\footnotetext{
${ }^{4}$ Notamment des programmes européens comme le Projet Pilote Urbain (PPU Bordeaux les deux rives) de 1997 à 2001, le Programme d'Initiative Communautaire Urban2 (PIC Urban2-Unicités) de 2001 à 2007, les Programmes Objectif 2 pour les périodes 1994-1999 et 2000-2006 et enfin, actuellement, un Programme Opérationnel Régional - FEDER de 2007 à 2013.
} 
dans le secteur central des quais, de la porte de Bourgogne aux Chartrons, la partie ouvrant sur les quartiers riches de la ville, mais l'ensemble des quais est en cours de rénovation. Si certains regrettent parfois une domination du minéral, à l'image de beaucoup de ville, là encore sous l'influence de codes et de normes représentant l'idée d'ordre et de "pureté », plusieurs parterres fleuris et des pelouses jalonnent tout de même cette étendue qui n'en possédait pas auparavant. Il y a en réalité un équilibre entre espaces "minéralisés » et "végétalisés", mais la dalle de granit de $3000 \mathrm{~m}^{2}$ du miroir d'eau en place centrale peut donner cette impression de domination de l'un sur l'autre, à l'image des grandes places rénovées dans Bordeaux, que ce soit la place de la Victoire, la place Pey-Berland ou encore la place de la Comédie qui ne sont agrémentées d'aucun espace de verdure.

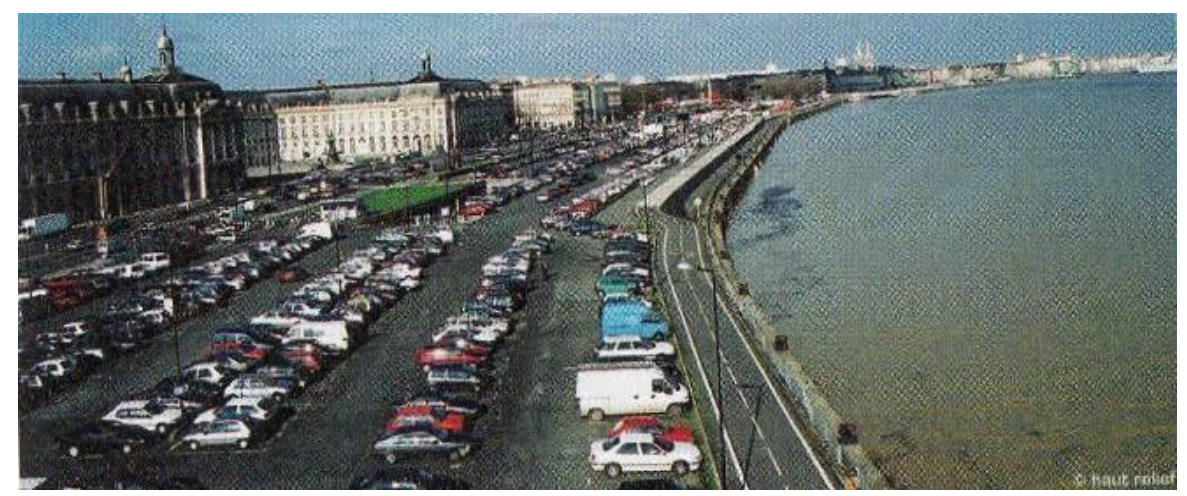

Les quais avant le réaménagement (source: CUB, DR)

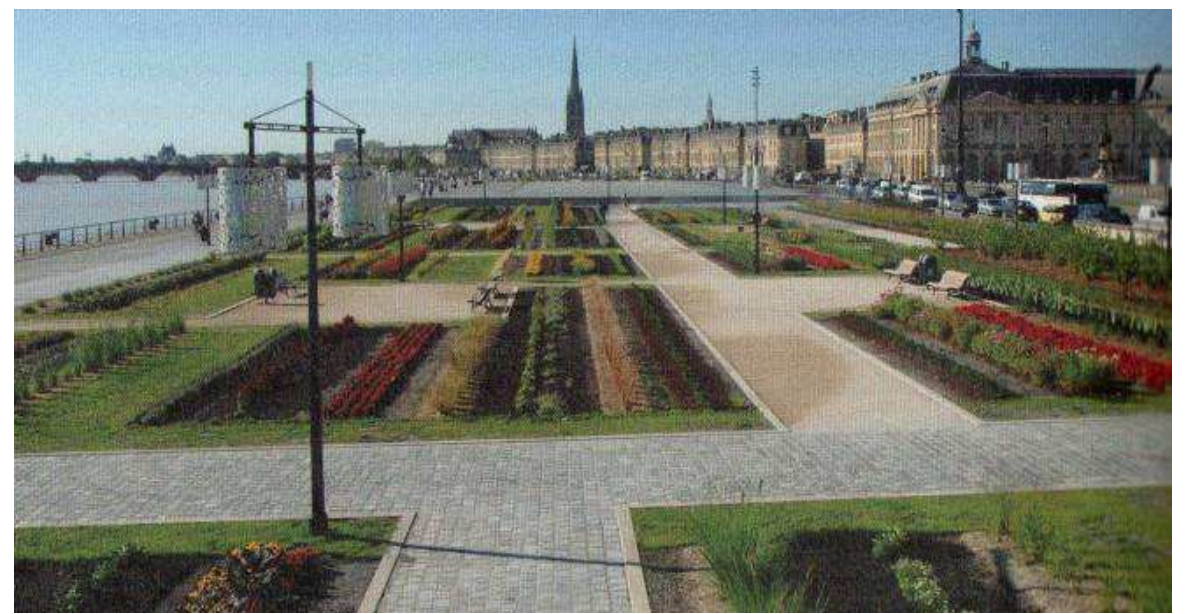

Les quais après le réaménagement (source : D.Doustin, M.Goudin, CUB, DR) 


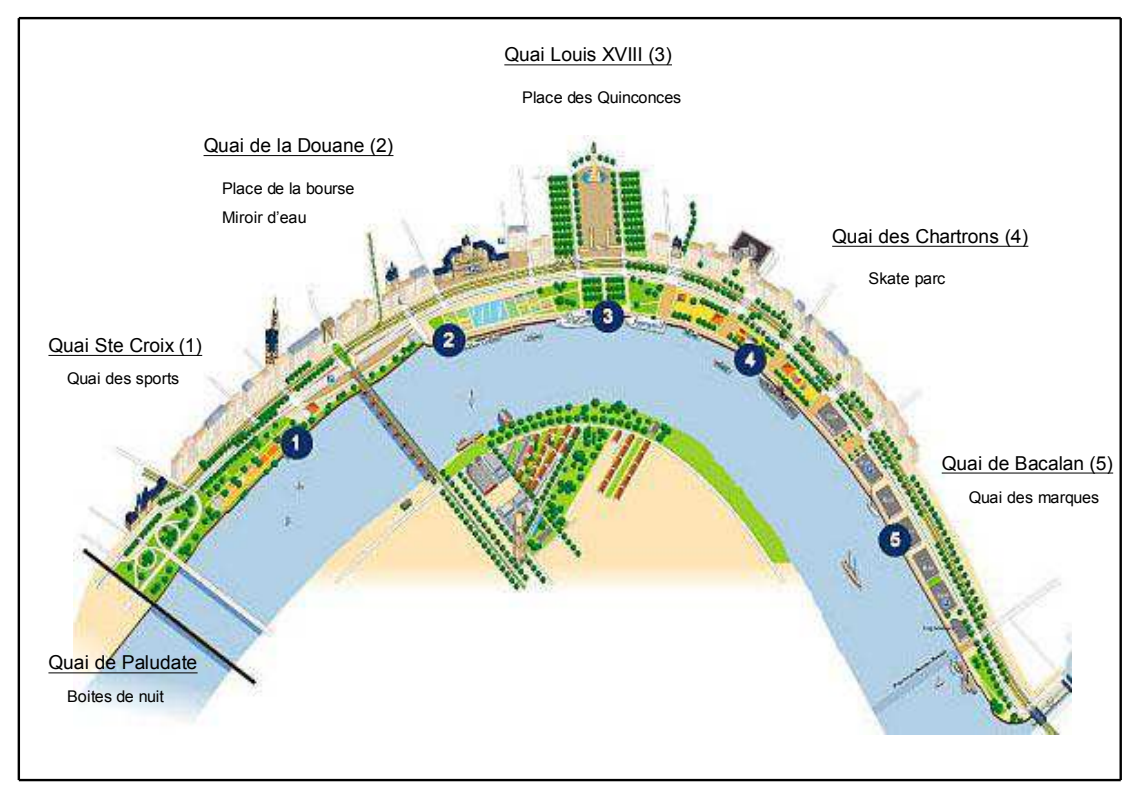

Plan et aménagement des quais, projet Courajoud

La première phase de la rénovation a été la mise en place du tramway de Ste Croix aux Quinconces. Dans cette partie, l'ensemble des façades XVIIIème a été nettoyé et le front de Garonne a été requalifié en promenade. Ce dernier est entièrement aménagé : équipé d'une piste cyclable, du miroir d'eau qui est depuis devenu célèbre et incontournable ${ }^{5}$ (face à la place de la Bourse), d'aires de jeux pour les enfants, d'installations sportives ainsi que de pelouses et plantations bien ordonnées.

Avec le prolongement de la ligne B du tramway jusqu'aux bassins à flots, la partie Nord des quais, le quai de Bacalan, est presque entièrement rénové. Au delà du skate parc, restent quelques travaux, mais la promenade est complètement aménagée en front d'eau, notamment grâce à la présence des galeries commerçantes installées dans les anciens hangars ${ }^{6}$.

Enfin, à l'extrémité opposée, au Sud, se trouve le quai de Paludate. Dans cette portion des quais située après le pont de chemin de fer, où le tramway ne passe pas et où il n'y a pas de commerces diurnes, les travaux d'aménagement sont en cours. Cette portion, en partie non classée par l'UNESCO et dernière phase du chantier des quais, est un secteur peu fréquenté le jour : il y a peu d'habitation et pas de commerce, les piétons y sont rares. En revanche, le passage des véhicules y est important du fait de la situation de cet axe entre les boulevards, la rocade, le centre ville et la rive droite. Si, durant la journée, le sud des quais est déserté par

\footnotetext{
${ }^{5}$ Le miroir d'eau de Bordeaux est le plus grand du monde, il s'agit d'une dalle de granit de plus de $3000 \mathrm{~m}^{2}$. En dehors de la période hivernale, les effets visuels changent : toutes les $15 \mathrm{mn}$ la dalle se recouvre de $2 \mathrm{~cm}$ d'eau créant un effet miroir où se reflètent les façades puis l'eau se retire et de la brume est envoyée par des buses, remplaçant l'effet miroir par un effet de brouillard. Le fontainier J.M Llorca s'est inspiré du phénomène naturel d'inondation de la place St Marc à Venise pour sa réalisation.

${ }^{6}$ Ces galeries sont appelées "quai des marques".
} 
les piétons, à l'inverse, la nuit, surtout passé minuit, il devient le centre de l'activité festive en raison de la présence de nombreuses boites de nuit.

On peut constater que l'ensemble de ces rénovations est surtout destiné aux activités diurnes ${ }^{7}$ et que les aménagements spécifiques à la nuit sont le fruit d'un urbanisme lumière mettant en scène et en avant certains lieux au détriment d'autres.

\section{2- LES CONSEQUENCES URBAINES DES RENOVATIONS}

\subsection{L'émergence de secteurs d'excellence : polarisation...}

A Bordeaux, le Plan lumière de la municipalité a coïncidé avec la mise en place du projet urbain ${ }^{8}$. "C'est à partir de l'effet choc de l'éclairage de la place de la Bourse, chef d'œuvre de la ville, que les mises en lumière se sont diffusées ॥ (J.Duc, 2000). On peut se demander pour quelles raisons une telle importance est accordée à l'illumination de ce lieu. Ce n'est certainement pas pour l'aspect pratique car ce n'est pas un quartier très passant en soirée ou la nuit ${ }^{9}$, il n'y a pas d'obstacles nécessitant plus de lumière pour le confort des piétons, et il ne s'agit pas non plus d'un endroit dangereux, " mal famé », où les zones d'ombres doivent être évitées pour la sécurité des passants. Quant à la route, elle est suffisamment éclairée pour les véhicules. Si ce lieu est tant éclairé et si bien mis en valeur, c'est pour son aspect esthétique. Nous convenons que l'esthétisme est nécessaire à l'attractivité d'un lieu qu'il s'agisse du rayonnement extérieur de la ville ou du bien-être des passants et des riverains et il faut noter que les bordelais sont fiers du patrimoine architectural d'exception de leur ville et qu'ils apprécient évidemment cette mise en lumière. Néanmoins, nous remarquons, ici, un choix politique dont l'intentionnalité se matérialise par la mise en scène de la façade. C'est ici, peut-être plus qu'ailleurs, que la ville est vue. D'où cette (sur)représentation du lieu dans les documents servant à la promotion de la ville.
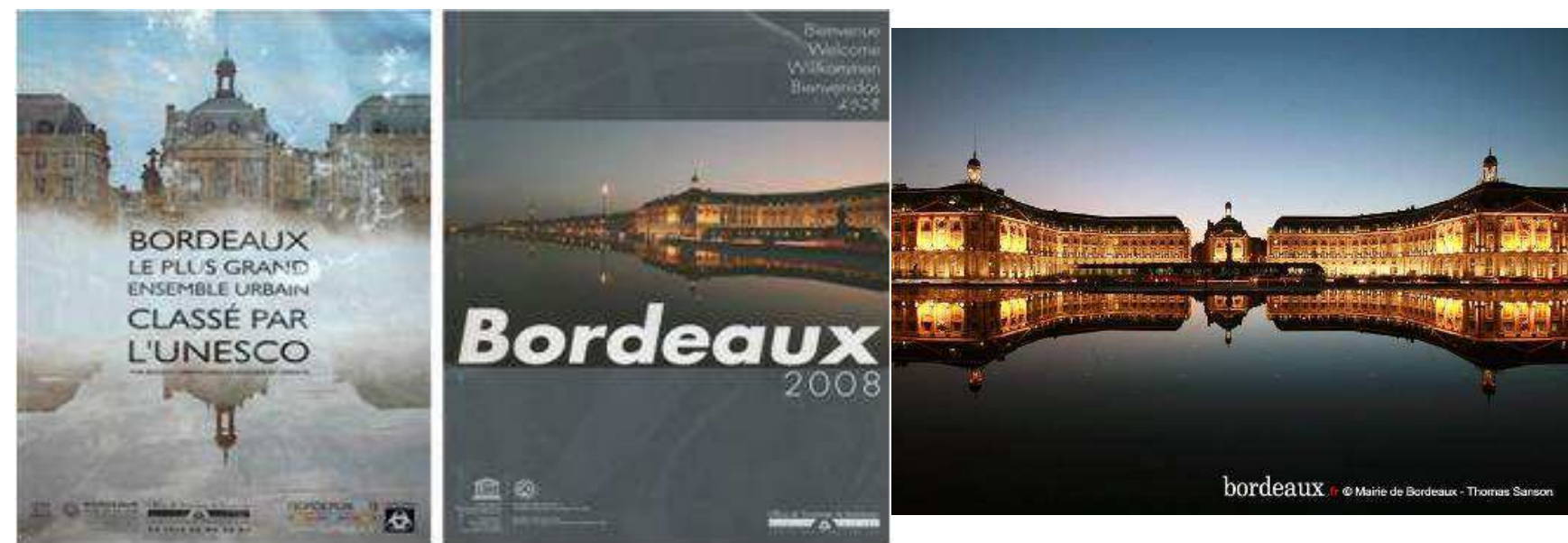

Affiches et brochures touristiques de la ville (source: Mairie de Bordeaux, office de tourisme, DR)

\footnotetext{
${ }^{7}$ Galeries commerçantes, jeux, promenade...

${ }^{8} \mathrm{~J}$. Duc, Le plan lumière de la ville de Bordeaux, mémoire de stage de $3^{\text {ème }}$ année à l'IEP de Bordeaux, 2000.

${ }^{9}$ Les restaurants et cafés à proximité sur les quais fermant tôt.
} 
Mais comme le rappelle Thierry Paquot (2006) en citant le point de vue d'Emile Magne dans L'esthétique des villes (1908) : " une rue, si belle soit-elle, ne manifeste pas d'existence par la seule vertu de son architecture. Organisme inerte, elle a besoin d'être habitée et parcourue pour acquérir une âme. ॥ Il est donc important de se demander quelle ville est construite, mise en scène, et pour qui ? Les priorités données aux rénovations et à la mise en valeur incitent à penser qu'elles sont destinées à une catégorie de population relativement aisée. En effet, ces embellissements favorisent la gentrification, voire l'embourgeoisement des riverains et concourent à la hausse des prix du foncier. Cette ascension ou "sélection" sociale de la rue peut également se lire à travers le type de commerces présents ${ }^{10}$ ou par le tarif élevé des consommations ${ }^{11}$.

La nomination de Bordeaux au patrimoine mondial de l'UNESCO et sa candidature à l'élection de capitale européenne de la culture en 2013 participent de cette logique que Philippe Dorthes (2007) qualifie de " politique de l'image ». Par le biais de politiques de la ville «embellissantes ॥, les municipalités, en suivant la mouvance générale de la mondialisation, ont leur part de responsabilité dans les inégalités en créant des "secteurs d'excellence " d'où sont exclues les populations les plus précaires, cette invisibilité ne cadrant pas avec le décor.

\section{2 ...et relégation}

Avec l'homogénéisation des villes et la normalisation des comportements, il y a de moins en moins de place pour les territoires ou les individus en marge des normes esthétiques ou sécuritaires édictées par la "société bien pensante » et dominante. Se pose alors le problème du droit à la ville pour les jeunes ${ }^{12}$ et les personnes marginalisées ${ }^{13}$. Ceux, entre autres, que Z. Bauman (2006) nomme les "déchets humains », ces exclus de la société qui suscitent la peur à cause d'un mode de vie parfois considéré comme déviant. Leur départ véhicule alors l'idée d'un quartier plus propre où le sentiment de sécurité est renforcé, ce qui le rend plus attractif. Les personnes et activités non gratae sont donc reléguées en périphérie, et ce d'une manière double : spatialement mais aussi temporellement, en étant reléguées en périphérie du jour, c'est-à-dire la nuit dans cet espacetemps où les normes diffèrent. Quant est-il de ces lieux laissés dans l'ombre, ceux que la municipalité ne veut pas montrer?

Lors d'une enquête réalisée entre août et novembre $2008^{14}$, nous avons pu constater la différence d'attention portée en matière de mise en lumière le long des quais. Nous avons choisi de décrire deux endroits peu éclairés et mis en valeur la nuit, et qui nous ont semblé représentatifs de l'action municipale et de sa

\footnotetext{
${ }^{10}$ Cabinets d'architectes, brocantes, banques...

${ }^{11}$ Il n'est pas rare payer 5 euros pour une bière quai des Chartrons (Enquête sur les tarifs des consommations dans les débits de boisson réalisée dans le cadre de la thèse -2008-).

${ }^{12}$ Par « les jeunes », nous entendons généralement une population âgée de 15 à 25 ans. Dans certains cas plus spécifiques, et/ou en rapport avec la législation, cette tranche d'âge peut varier : pour les boites de nuit par exemple, il s'agit des 18-30 ans.

${ }^{13}$ Cf. Gwiazdzinski, Deleuil, Jouenne, Bauman...

${ }^{14}$ Travail d'observation effectué dans le cadre de la thèse.
} 
volonté à orienter les pratiques des usagers. Le premier marque une relégation périphérique, le second une relégation temporelle.

Le premier exemple que nous avons choisi de mettre en avant est le quai de Paludate. Situé à l'extrémité sud des quais, derrière la gare, c'est le lieu de concentration des pratiques nocturnes, parfois transgressives (prostitution, forte consommation d'alcool, agressions...), rassemblant la plupart des discothèques de la ville. Dans ce secteur, déserté le jour et pourtant très fréquenté la nuit, seuls quelques lampadaires et les néons des boites de nuit apportent un éclairage discret. Cette relative obscurité convient aux prostituées à proximité mais elle réduit l'attractivité du lieu. Si les noctambules qui arrivent généralement en groupes à la fermeture des bars pour se rendre en discothèque ont rarement ce sentiment d'insécurité (on peut penser que l'alcool inhibe la peur), en revanche, ce faible éclairage rebute parfois les filles qui peuvent se sentir obligées d'être accompagnées par des amis de sexe masculin ${ }^{15}$. On constate, dans ce cas, que la rue est plus masculine que féminine ${ }^{16}$. Concernant l'aspect dangereux parfois cité par les usagers, il n'est pas qu'imaginaire, puisque le quai de Paludate est le lieu où le nombre d'interpellations réalisées par les policiers est le plus important, en particulier les soirs de week-end. ${ }^{17}$

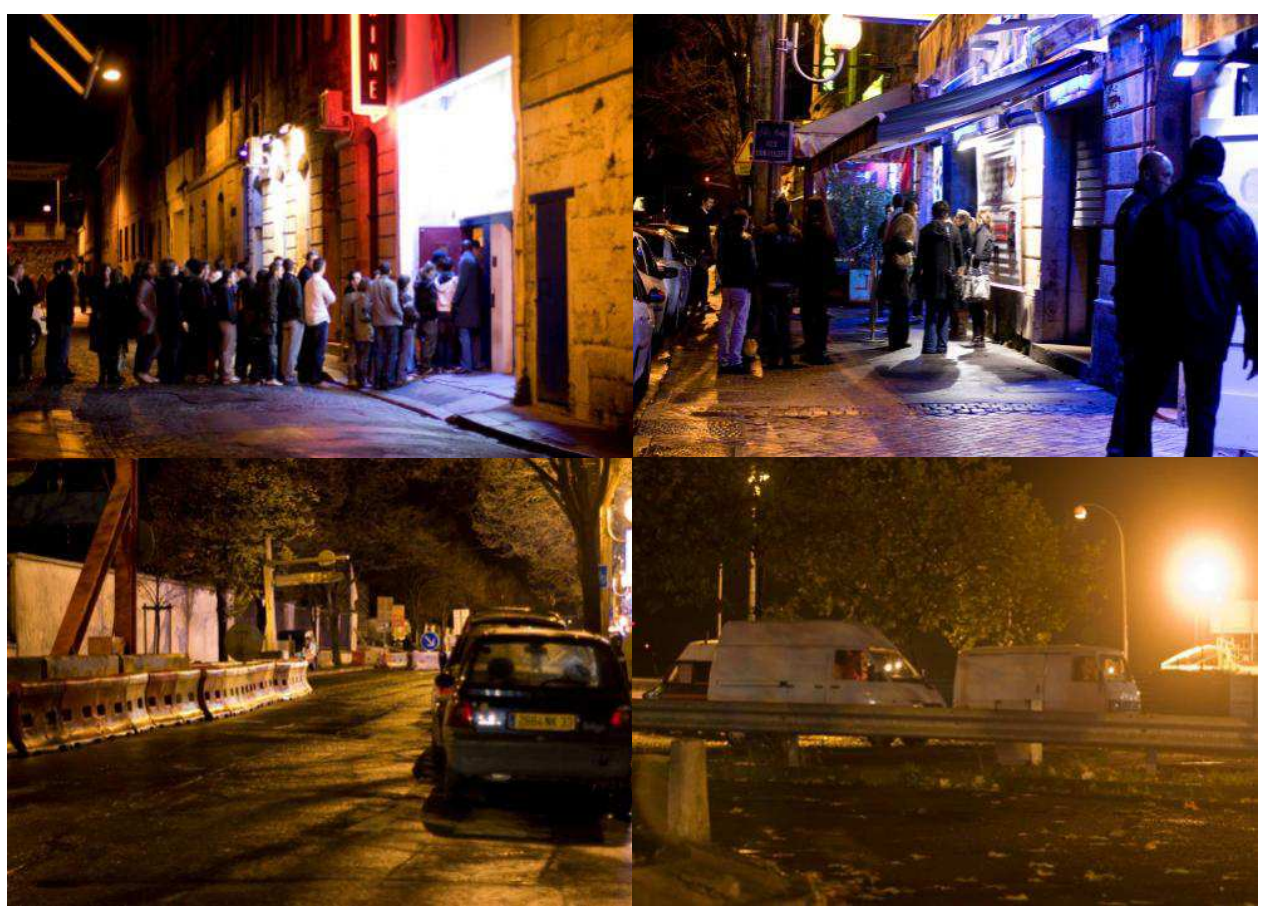

Le quai de Paludate : boites de nuit, travaux et prostitution (Clichés : J. Cazzulo).

\footnotetext{
${ }^{15}$ Entretiens réalisés en 2008.

${ }^{16}$ Voir les travaux d'Yves Raibaud à ce sujet.

${ }^{17}$ Entretiens réalisés auprès de la brigade des débits de boisson et du proxénétisme et de la brigade anti-criminalité de Bordeaux, mai 2009.
} 
Le second exemple est le skate parc situé quai des Chartrons, au Nord, un des riches quartiers de la ville. Cet équipement destiné aux jeunes ${ }^{18}$ est très peu éclairé la nuit et, de fait, l'utilisation des installations n'est autorisée que jusqu'à 22h. Pour quelles raisons les jeunes ne pourraient-ils pas pratiquer le skate après cette heure? On pourrait avancer différents arguments pour expliquer cette interdiction et donc le manque de lumière: les nuisances sonores (et la fameuse limite des $22 \mathrm{~h}$ pour le tapage nocturne); la sécurité, les dégradations...mais aucun ne s'avèrent vraiment justifiés: le bruit ne semble pas atteindre les habitations situées de l'autre coté de la rue et ce lieu n'est pas surveillé en journée non plus. Si l'on se réfère aux travaux de Luc Gwiazdzinski, spécialiste de la nuit, l'argument sécuritaire n'est pas valable, car c'est en investissant la nuit qu'elle devient plus sûre. En fait, les pratiques sportives urbaines telles que le skate ou le roller commencent tout juste à être acceptées en ville mais pas au point de laisser leurs adeptes faire du "street ॥ c'est-à-dire d'utiliser le mobilier urbain comme aire de pratique. C'est pour cela que des efforts sont fait dans de nombreuses villes en matière d'aménagements spécifiques, afin de canaliser et de contrôler ces pratiques sportives parfois considérées comme transgressives. Cette acceptation de la pratique du skate en ville, bien qu'encadrée et limitée au jour, est déjà un premier pas pour la municipalité qui n'est pas prête à autoriser cette pratique la nuit. En effet, on n'imagine pas les autorités permettre une pratique encore considérée comme transgressive par certains, dans une temporalité encore parfois perçue comme subversive.

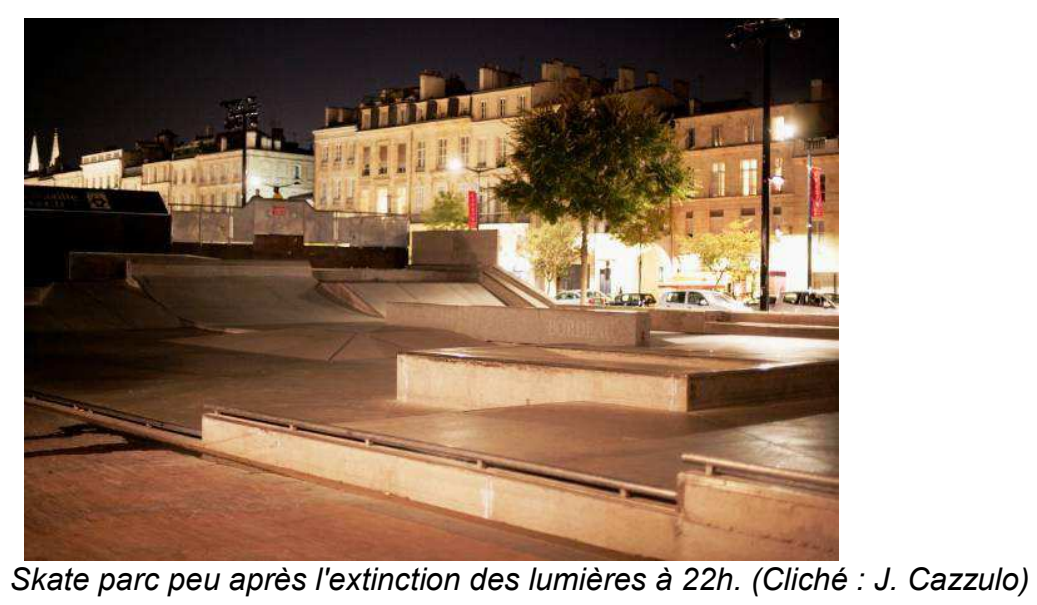

Les deux lieux que nous venons de présenter sont des endroits attractifs pour les jeunes, le premier uniquement la nuit du fait du type d'établissements présents. Le second pourrait l'être autant le jour que la nuit, mais dans les deux cas, la municipalité ne met pas leurs environnements respectifs en valeur la nuit. Ces situations font dire à Philippe Dorthes (2007) concernant Bordeaux « qu'il y a une carence évidente d'équipements spécifiques qui permettraient à la jeunesse de se regrouper, de s'exprimer, de s'amuser. » et d'ajouter que : «les citoyens sont des figurants tout juste bons à peupler les quais aux jours de "fête " jusqu'à ce que la police culturelle les invite impérieusement à se disperser sur le coup de minuit. ».

\footnotetext{
${ }^{18}$ Une aire « débutant » est à la disposition des enfants de 3 à 10 ans qui doivent être accompagnés, le reste du Skate Parc est accessible à partir de 8 ans. Le soir, les pratiquants ont entre 15 et 25 ans en moyenne.
} 


\section{3 - LES QUAIS : UN SYSTEME SPATIO-TEMPOREL}

\subsection{L'attractivité des quais}

Axe choisi pour notre étude, les quais ne sont pas une rue "classique", témoins du passé portuaire de la ville, il s'agit d'une bande de $80 \mathrm{~m}$ de large entre façades et Garonne et sur 4,5 km de long de la gare St Jean aux bassins à flots. Dans le cadre des rénovations, de nouvelles fonctions ont été attribuées à des espaces qui n'en avaient plus depuis plusieurs années. Si les quais ont été très attractifs durant des siècles grâce aux activités portuaires, depuis le départ de ces activités à la fin des années 1980 ils étaient laissés à l'abandon, sans âme et véhiculaient une image négative. Cette mauvaise réputation des quais avait contribué à l'augmentation du nombre de logements vacants, à la paupérisation et à la hausse de l'insécurité ${ }^{19}$. Redonner une fonction à ces espaces les a rendus de nouveau attractifs: ils attirent populations locales et touristes, ne sont plus synonymes d'insécurité, et les logements à proximité font l'objet d'une forte demande et de loyers en hausse. S'il existe des rues plus symboliques que d'autres, on assiste à la mutation de certaines rues qui peuvent devenir emblématiques, ou perdre ce statut, comme ce fut le cas des quais qui connurent leurs heures de gloire, puis la désaffection avant de redevenir un des lieux de promenade privilégiés des bordelais et des touristes. Ainsi, l'article de Marie-Françoise Fleury et Hervé Théry (2005) propose de réviser le nom des rues dans le Monopoly afin de "faire du plateau de jeu une image plus fidèle du Paris d'aujourd'hui ».

De la même façon à Bordeaux, la rénovation des quais a été voulue par la municipalité afin de rendre attractive la ville en mettant en avant l'esthétique architecturale de cette vitrine et en proposant des fonctions nouvelles. Les zones de loisir, de chalandise et de restauration qui sont principalement au centre géométrique des quais sont très attractives la journée. La nuit, la mise en lumière de cette partie centrale plait aux touristes et aux passants qui rentrent chez eux. Il arrive aussi que, le soir, la ville organise des événements comme la fête du fleuve ou la fête du vin qui ont lieu tous les ans à tour de rôle, ainsi que des bals durant l'été. Ces manifestations se situent toujours au centre, sur la place des Quinconces ou aux alentours, et attirent une foule importante. Mais comme nous le faisions remarquer précédemment en citant $P$. Dorthes, l'orchestre s'arrête de jouer à minuit, parfois dès $23 \mathrm{~h}$ comme c'est le cas des soirées dansantes organisées au quai des sports les samedis de mi-juillet à mi-août, ensuite la foule se disperse. Après cette heure symbolique, il ne reste que le décor, le centre n'est plus attractif. Les lumières s'éteignent ou se tamisent sur les lieux dédiés aux activités sportives (extinction des lumières à $22 \mathrm{~h}$ au quai des sports et au skate parc), les lieux de restauration ferment et les bâtiments s'illuminent. L'attractivité se réduit aussi de fait par l'arrêt des transports en commun, bus et trams du soir terminent leurs services aux alentours d'une heure du matin. Cet horaire à un aspect discriminant, car si le réseau de transport offre une assez bonne couverture spatiale de la ville centre et des communes alentours, au-delà d'une heure du matin les personnes n'habitant pas dans le centre ville mais ne désirant

\footnotetext{
${ }^{19} \mathrm{Cf}$. le programme opérationnel Urban-Unicités, 2001.
} 
pas rentrer sont obligés d'avoir une voiture, de prendre un taxi ou de marcher. Un seul bus fonctionne les nuits du jeudi au samedi, il s'agit du Sll qui relie la gare St Jean au campus universitaire de Pessac en passant par la place de la Victoire. C'est donc entre minuit et 2 heures que le basculement se fait et que la périphérie prend le relais. En effet, avant minuit les boites de nuit situées quai de Paludate sont presque vides, et les clients arrivent passée cette heure et surtout à partir de $2 \mathrm{~h}$ du matin, à la fermeture des bars.

\subsection{Un modèle centre-périphérie qui s'inverse la nuit}

La journée, le soleil éclaire la terre en ne privilégiant aucun point particulier, à la différence de la lumière naturelle du jour, la lumière artificielle de la nuit n'est pas uniforme, son intensité varie selon les lieux et dépend du choix délibéré des autorités. Ces dernières participent ainsi à la mise en valeur esthétique du centre des quais et tentent de faire ignorer la périphérie sud de cet axe en la laissant dans la pénombre. En France, La nuit reste une frontière, un espace très peu colonisé par les activités économiques si ce n'est par des activités spécifiques à cette temporalité (bars, boites, prostitutions...). Elle est encore aujourd'hui considérée comme une marge du jour, et est très peu prise en compte dans les projets d'aménagement. Pourtant, cette marge du jour existe et s'exprime en marge du centre pour ceux qui la pratiquent. La nuit les sentiments sont souvent exacerbés, comme la peur ou l'excitation, particulièrement à l'extérieur, en dehors du cocon protecteur de l'habitation. Et si le sentiment de liberté est plus grand durant les heures sombres, cette idée est en partie un leurre puisque le champ des activités possibles est fortement réduit. D'une part, car comme partout en France, à de rares exceptions près, la plupart des commerces et des lieux de loisirs sont fermés. Mais d'autre part, car la municipalité limite l'accès aux lieux de loisirs qui sont ou qui pourraient être ouverts la nuit en ignorant cet espacetemps qui fait partie de la ville au même titre que le jour. Certes, la ville est mise en valeur la nuit par un urbanisme lumière sophistiqué, mais à seule visée esthétique puisque l'offre nocturne est très réduite. Ce qui vaut d'ailleurs à la ville de Bordeaux le surnom de "belle endormie ". Cet axe, symbole fort de la ville est, comme nous l'avons vu, l'objet d'une attention particulière, notamment ces dernières années avec l'introduction du tramway et l'ensemble des projets d'aménagement mis en œuvre par la municipalité. Néanmoins, on peut regretter que cette attention se limite au jour et au centre des quais, partie " riche " de la ville et se contente, la nuit, de mettre en scène un décor somptueux mais où seuls les monuments comptent, les acteurs n'étant que rarement conviés.

Les quais de Bordeaux peuvent alors être considérés comme un système spatial auquel il est possible d'appliquer le modèle centre-périphérie (Grataloup, 2004). En effet, comme nous l'avons vu précédemment, on retrouve d'une part, une centralité autour de la place de la Bourse et des Quinconces marquée par la concentration de population, d'activités et une forte attractivité. Et d'autre part, une périphérie reléguée et s'inscrivant en négatif par rapport au centre, le quai de Paludate délaissé des aménageurs. Une des spécificités des quais est que la polarité s'inverse entre le jour et la nuit. En journée, le centre névralgique de cet axe est le centre géographique, tandis que les extrémités sont moins fréquentées. 
A l'inverse, la nuit, le centre géographique des quais est peu animé tandis que les " bouts des quais » se réveillent. Cela est dû à la répartition des activités : les bars, les boîtes de nuit, les clubs et les commerces ouverts la nuit sont situés majoritairement aux extrémités des quais, notamment du côté du quai de Paludate. Ce dernier draine plus de monde la nuit du fait d'une offre de service plus nombreuse, plus diversifiée et adaptée à la demande des noctambules. L'étude des pratiques et de la mise en valeur des quais à Bordeaux nous a invité à une réflexion sur le modèle centre-périphérie et sur son inversion à la faveur de la nuit. Celle-ci est liée au mouvement de balancier jour-nuit et met en évidence l'aspect temporel de l'attractivité. On assiste ainsi à une inversion du modèle centre-périphérie entre le jour et la nuit sur les quais.

La nuit pourrait elle-même être vue comme l'un des deux éléments du modèle centre-périphérie : si l'on considère que le modèle s'applique, en théorie, à un système spatial, on peut aussi voir le jour comme un système, une entité temporelle où le jour et la nuit seraient les deux éléments constitutifs du même objet (Saunier, 1999). Dans ce cas, la journée serait le centre et la nuit la périphérie. Le modèle centre-périphérie appliqué à ce couple jour/nuit prend sens par la relation dialectique et dissymétrique qui les unit (Catan, 2006). Il est alors pertinent, dans le cas précis de l'étude des quais, de coupler ces deux systèmes que sont d'une part, les quais et d'autre part, la journée. On peut alors faire un parallèle entre les centres: celui du système spatial (la place de la Bourse) et celui du système temporel (le jour), ces centres étant dominants, ainsi qu'entre les périphéries (le quai de Paludate et la nuit), ces dernières tendant à être reléguées. En se plaçant du point de vue des pratiques nocturnes, les polarités s'inversent et les périphéries s'imposent.

\section{CONCLUSION}

Pour conclure, si la rue donne le ton et la couleur d'une ville à une époque donnée (Farge, 1979,1992) les quais donnent actuellement de Bordeaux l'image d'une ville " bourgeoise » et aseptisée. Certes, la ville de Bordeaux a toujours été une ville bourgeoise, mais depuis la fin des activités portuaires ce segment urbain délaissé ne "collait" pas avec l'image globale de la ville. Grâce aux rénovations, les quais qui font office de vitrine urbaine donne d'emblée à voir l'identité locale fondée sur le fleuve et le patrimoine architectural. Nous pouvons dire que la mondialisation, par le biais de la concurrence entre les villes, a pour incidence une politique de la ville visant à " nettoyer " certains secteurs de la ville dont les quais. La standardisation des quais, en réponse à des normes hygiénistes et sécuritaires ou découlant d'un certain imaginaire éco-sanitaire, participe à faire de cet axe un symbole et une vitrine de la ville, notamment par la mise en valeur d'une grande portion dont le point d'orgue est la place de la Bourse. Cette homogénéisation morpho-sociologique est visible dans l'uniformisation du paysage urbain ainsi que dans les pratiques des usagers. Par les rénovations d'envergure, la municipalité a mis en scène un somptueux décor dans lequel les individus doivent suivre le scénario prescrit. Mais la ville reste, malgré les contraintes imposées, un espace vécu où les marges (sociales et spatiales) arrivent encore à jouer un rôle en existant pour une partie de la nuit. 
Ainsi, l'émergence de secteurs d'excellence va de pair avec une relégation périphérique créant des discontinuités le long de cet axe. Ce dernier élément nous a invité à une réflexion sur l'inversion du modèle centre-périphérie entre le jour et la nuit. En effet, dans ce système spatial formé par les quais, le centre géographique est mis en valeur par des aménagements à visée diurne, il est donc dominant le jour et les extrémités délaissées sont reléguées. Mais l'attractivité nocturne de cette périphérie géographique fait de cette marge délaissée des aménagements le centre actif des quais, tandis que la place de la Bourse, si bien mise en lumière la nuit, joue seulement le rôle d'un tableau ou d'une scène sans acteurs.

\section{REFERENCES}

Bauman, Z. (2004). Vies perdues. La modernité et ses exclus. Paris: Payot \& rivages.

Bernié-Boissard, C. (2008). Des mots qui font la ville. Paris: La dispute.

Bidou-Zachariasen C. (dir.) (2002). Retours en ville, des processus de "gentrification" urbaine aux politiques de "revitalisation" des centres. Paris : Descartes et Cie.

Cattan, N. (2007). http://hal.archives-ouvertes.fr/docs/00/16/25/80/PDF/Centre-Peripheriedicodef.pdf.

Deleuil, J.M. (1994). Lyon la nuit. Lyon : Presses Universitaires de Lyon.

Dorthes, P. (2007). Bordeaux mode de ville. Bordeaux: pleine page.

Duc, J. (2000). Le plan lumière de la ville de Bordeaux. Mémoire de stage de $3^{\text {ème }}$ année à l'IEP de Bordeaux.

Farge, A. $(1979,1992)$. Vivre dans la rue à Paris au XVIIIème siècle. Paris: Gallimard (1979) Folio (1992).

Fleury M.F et Théry H. (2005). Les rues de Paris vues par le monopoly, une proposition de révision. Mappemonde $\mathrm{n}^{\circ} 77$. http://mappemonde.mgm.fr/num5/articles/art05104.html.

Gasnier, A. (2006). Entre privatisation des lieux publics et publicisation des lieux privés. Revue Urbanisme, 346 (Espaces publics), p.70-73.

Grafmeyer, Y. J., Isaac. (2004). L'école de Chicago. Naissance de l'écologie urbaine. Paris: Flammarion.

Grataloup, C. (2004). http://www.hypergeo.eu/spip.php?article10.

Gwiazdzinski, L. (2002). La ville 24 heures sur 24. La Tour d'Aigues: éditions de l'aube. 
Gwiazdzinski, L. (2006). Les marges peuvent devenir des cœurs. Revue Territoires, 467, p.16-18.

Gwiazdzinski, L. (2007). Nuits d'Europe. Pour des villes accessibles et hospitalières. Belfort: Université de technologie de Belfort-Montbéliard (UTBM).

Jacobs J. (1991). Déclin et survie des grandes villes américaines. Lièges : Mardaga.

Jouenne, N. (2007). De la contorsion de l'espace public au déni de la personne ou comment se débarrasser proprement des sans logis. Paper presented at the Rencontres Thomas More. 24, 25 mars 2007. De l'effacement de la personne.

Jouenne, N. (2008). Contresens du design dans l'espace public. http://halshs.archivesouvertes.fr/halshs-00248912.

Paquot, T. (2006). Des corps urbains. Sensibilités entre béton et bitume. Paris: Autrement.

Programme opérationnel Unicités-urban2. (2001). http://www.urban-unicites.com.

Roulleau-Berger, L. (2004). La rue, miroir des peurs et des solidarités. Paris: PUF.

Saunier, G. (1999). Quelques réflexions sur le concept de Centre et Périphérie. Hypothèses. p. $175-180$

Sennett, R. (2009). La conscience de l'œil. Paris : Verdier. 